\title{
Nonlinear wave structures in collisional plasma of auroral E-region ionosphere
}

\author{
A. V. Volosevich ${ }^{1}$, Yu. I. Galperin ${ }^{2}$ \\ 1 Mogilev Pedagogical Institute, Mogilev, 212026, Belarus Republic \\ 2 Space Research Institute of RAN, Moscow, 117810, Russia
}

Received: 17 April 1996/Revised: 23 July 1996/Accepted: 30 July 1996

\begin{abstract}
Studies of the auroral plasma with small-scale inhomogenieties producing the VHF-radar reflections (radar aurora) when observed in conditions of the saturated Farley-Buneman instability within the auroral E region, show strong nonlinear interactions and density fluctuations of $5-15 \%$. Such nonlinearity and high fluctation amplitudes are inconsistent with the limitations of the weak turbulence theory, and thus a theory for arbitrary amplitudes is needed. To this end, a nonlinear theory is described for electrostatic MHD moving plasma structures of arbitrary amplitude for conditions throughout the altitude range of the collisional auroral $\mathrm{E}$ region. The equations are derived, from electron and ion motion selfconsistent with the electric field, for the general case of the one-dimensional problem. They take into account nonlinearity, electron and ion inertia, diffusion, deviation from quasi-neutrality, and dynamical ion viscosity. The importance of the ion viscosity for dispersion is stressed, while deviation from the quasi-neutrality can be important only at rather low plasma densities, not typical for the auroral E region. In a small amplitude limit these equations have classical nonlinear solutions of the type of "electrostatic shock wave" or of knoidal waves. In a particular case these knoidal waves degrade to a dissipative soliton. A two-dimensional case of a quasi-neutral plasma is considered in the plane perpendicular to the magnetic field by way of the Poisson brackets, but neglecting the nonlinearity and ion inertia. It is shown that in these conditions an effective saturation can be achieved at the stationary turbulence level of order of $10 \%$.
\end{abstract}

\section{Introduction and problem statement}

Space experiments with high time/space resolution have shown the appearance of nonlinear wave-like, or soliton-

Corresponding author: Yu. I. Galperin type structures in the auroral plasma at magnetospheric altitudes. The examples are the data from the satellites S3-3 (Temerin et al., 1979, 1981), Viking (Koskinen et al., 1990) and Freja (Erlandson et al., 1994) where the plasma can be considered as collisionless.

Several groups are now developing theories and/or numerical models to describe such collisionless plasma structures (see, for example, Schamel, 1986; Tetreault, 1991; Seyler, 1990; Chang, 1992; Romero et al., 1992). Volosevich and Galperin (1995), in the frame of one-dimensional nonlinear MHD stationary wave theory, modified and generalized the approach by Temerin et al. (1979) and Chang (1982). The authors reached a qualitative agreement with the satellite experimental data on all the observable parameters of the so-called "ion hole" structures. In Volosevich et al. (1995) the theory was generalized to the collisional auroral E-region plasma. This approach will be further extended here. New effects appear for its two-dimensional versions (as will be discussed).

At auroral ionosphere altitudes where collisions generally may not be neglected, there were several very interesting rocket experiments that also indicated the existence of nonlinear phenomena, wave bursts, and impulsive particle acceleration (see Reme and Bosqued, 1971; O'Brien and Reasoner, 1971; Kelley and Mozer, 1973; Bahnsen et al., 1978, Whalen and Daly, 1979, Boehm et al., 1984; LaBelle et al., 1986; McFadden et al., 1986; Bryant et al., 1991; Arnoldy et al., 1992; Kintner et al., 1992; Rinnert, 1992). In the collisional auroral E-region plasma some rocket experiments and phenomena of radar aurora indicated the existence of plasma density inhomogenieties with amplitudes up to $7-10 \%$ and scales in the decimeter and meter range [see Kelley and Mozer (1973), Bahnsen et al. (1978), and a reevaluation in Primdahl and Bahnsen (1985)]. However, these E-region phenomena are still not well studied experimentally from rockets to measure their waveforms and lifetimes.

For more than three decades the Farley-Buneman (FB) instability has been considered the generation mechanism for the E-region inhomogenieties responsible for the 
VHF-radar aurora (see reviews by Fejer and Kelley, 1980; Haldoupis, 1989). In most of these theories of the E region, a linear theory was used for the instabilities leading to the plasma inhomogeniety formation, for example, threewave interactions, which were considered weak. Also, the FB wave dispersion and ion viscosity were mostly neglected. Studies of the linear stage for this instability were indeed successful in the interpretation of many characteristics of the radar aurora.

Meanwhile, the theory for the nonlinear stage still meets with some important difficulties and contradictions. One of the reasons for this, from our point of view, is that linearly growing waves in the theory have no dispersion, and thus grow to rather high amplitudes. At such amplitudes nonlinear interactions cannot be considered as weak, and the main condition for applicability of the weak turbulence theory is violated.

Within the frame of the nonlinear models by Volosevich (1978), Hamza and St.-Maurice (1995), and others, the wave-wave interactions (three-wave as well as four-wave) lead to nonlinear frequency shifts. These shifts, in their turn, decrease the linear increments and stabilize the instability at a certain (rather low) stationary level.

However, there is a principal limitation in the usually applied linear theory. When kinetic effects are taken into account, such as Landau damping on ions, the range in wave vectors $\vec{k}$ of the excited waves becomes limited. This means that realistic FB waves must have a dispersion. As was shown in Volosevich (1978), the Landau damping on ions, in the dispersion relation for the FB waves, is equivalent to taking into account the ion dynamical viscosity in the hydrodynamic model by Gershman et al. (1976) and Volosevich (1978). Thus the dispersion relation may be written as

$$
\omega=\frac{\vec{k} \cdot \vec{V}_{o e}}{1+\hat{R}\left(1+g^{2} \frac{k^{2} U_{T i}^{2}}{v_{i}^{2}}\right)} ; \quad \hat{R}=\frac{v_{i} v_{e}}{\omega_{H i} \cdot \omega_{H e}} \cdot\left(1+\frac{k_{\|}^{2}}{k_{\perp}^{2}} \cdot \frac{\omega_{H e}^{2}}{v_{e}^{2}}\right) ;
$$

where $k_{\|}, k_{\perp}$ are wave vector $\vec{k}$ components along and perpendicular to the magnetic field, $U_{T i}=\left(T_{i} / m_{i}\right)^{1 / 2}$ is the ion temperature, $v_{i}, v_{e}$ are collision frequencies of ions and electrons, $\omega_{\mathrm{Hi}}, \omega_{\mathrm{He}}$ are the respective gyrofrequencies, and $g^{2}$ is a dimensionless coefficient of order 1 to 3 related to a particular hydrodynamic model. It follows that the wave dispersion is inherent to the realistic FB waves, and this property is very important for their nonlinear dynamics.

In some papers a predetermined relation was supposed between the plasma electron density $n_{e}$ and electrostatic potential $\varphi$ : either a linear, or the Boltzmann one.

However, in Volosevich and Galperin (1995) a more general relation between the density and the electric potential was derived for any charged particle population which allows to study various effects including a loss of quasi-neutrality. The resulting electric field can be found from the Poisson equation, or from the quasi-neutrality condition, and it is self-consistent with the particle motion within the finite-amplitude plasma structure that was considered.

One of the principal problems for both collisional and collisionless nonlinear MHD equations for auroral re- gions, is the way to avoid the singularity in the solutions; that is, the wave overturn. What are the conditions and effects needed for a stabilization of nonlinear structures at a finite amplitude? These effects will define the space and frequency scale for a particular type of nonlinear wave structure. Obviously, only such durable, quasi-steady structures have chances to be observed from a low-altitude satellite or a rocket. Or, if the conditions for stabilization are not met, only a highly turbulent plasma will result from linearly growing and overturning waves.

We consider what conditions are necessary (but not generally sufficient) to reach stabilization at some finite level for a wave-like or a soliton-like nonlinear structure. We shall evaluate its corresponding scales in space and time for a simplified range of conditions (Sect. 2). A relative importance of the ion Landau damping and quasineutrality condition for the corrections to the dispersion of the FB waves is considered in Sect. 3. Some new effects will be considered for a two-dimensional generalization of the theory (Sect. 4). Discussion of results, including the problem of comparisons with available experimental data, will be briefly considered in Sect. 5. Conclusions are presented in Sect. 6.

\section{Theoretical Model}

The evolution of nonlinear structures will be described by a system of quasi-hydrodynamic equations of motion of charged particles of type $\alpha$ ( $\alpha=e$ for electrons, $\alpha=i$ for ions) together with the continuity equation and Poisson equation for the electric field:

$$
\begin{aligned}
& \frac{\partial \vec{U}_{\alpha}}{\partial t}+\left(\vec{U}_{\alpha} \cdot \vec{\nabla}\right) \vec{U}_{\alpha}=-\vec{\nabla} \Phi_{\alpha}+\omega_{H \alpha}\left[\vec{U}_{\alpha}, \vec{e}_{z}\right]-v_{\alpha} \vec{U}_{\alpha}-\vec{\Gamma}_{\alpha} ;(1) \\
& \frac{\partial n_{\alpha}}{\partial t}+\operatorname{div}\left(n_{\alpha} \vec{U}_{\alpha}\right)=0 ; \\
& \nabla^{2} \varphi=\frac{e}{\varepsilon_{o}}\left(n_{e}-n_{i}\right) ;
\end{aligned}
$$

where:

$\Phi_{\alpha}=\frac{e_{\alpha}}{m_{\alpha}} \varphi+U_{T \alpha}^{2} \ln \left(n_{\alpha}\right) ; \quad \vec{\Gamma}_{\alpha}=\eta_{\alpha}\left(\Delta \vec{U}_{\alpha}+\frac{1}{3} \vec{\nabla} \cdot\left(\vec{\nabla} \vec{U}_{\alpha}\right)\right) ;$

electric field is supposed to be electrostatic $\vec{E}=-\nabla \varphi ; \eta_{\alpha}$ is the coefficient of ion dynamical viscosity (Braginsky, 1963; Gershman et al., 1976). Other definitions are standard:

$\omega_{H a}=e_{a}\left|\vec{B}_{o}\right| / m_{a} ; \quad \vec{B}_{o}=\left|\vec{B}_{o}\right| \cdot \vec{e}_{z} ; \quad U_{T a}^{2}=2 k T_{a} / m_{a} ;$

and $T_{\alpha}, m_{\alpha}, e_{\alpha}, U_{\alpha}, n_{\alpha}$, and $v_{\alpha}$ are the temperature, mass, charge, velocity, number density, and collision frequency (or, an effective collision frequency) of particles of the sort $\alpha$.

In this paper we shall consider the nonlinear interactions as strong, and use the strong turbulence theory for their description. Firstly, we shall study possible roles of various factors for the development of quasi-stationary nonlinear structures such as nonlinearity, inertia, collisions, dynamical viscosity, and deviations from quasi-neutrality. 
We consider here a strongly collisional plasma corresponding to the E region of auroral ionosphere ("dynamoregion"). In this region electrons are magnetized $v_{e} \ll \omega_{H e}$, while ions are nonmagnetized $v_{i}>\omega_{H i}\left(v_{e}, v_{i}, \omega_{H i}, \omega_{H e}\right.$ are collisional frequencies of electrons and ions and their gyrofrequencies).

As is known, the primary reason for the low-frequency instabilities in the auroral E region is the electric field and currents imposed by the magnetosphere, and ionospheric currents and fields induced thereby. The instabilities can generate plasma inhomogenieties and small-scale structures, which are able to scatter radiowaves up to VHF frequencies (so-called radar aurora). The exact nature of the radar aurora scatterers is poorly known, despite great efforts of various groups, due to the extreme complexity of the problem and its dependence on various parameters of the medium which need to be taken into consideration for the experimental data comparisons with theory.

Our aim here is to propose a theoretical framework for such comparisons which could be applicable to nonlinear, collisional, strongly turbulent, and dynamic auroral Eregion plasma at different altitudes. We shall start from a one-dimensional model, and later will briefly consider two-dimensional effects in a simplified model.

\section{One-dimensional model}

\subsection{General equations}

We shall consider here quasi-stationary electrostatic nonlinear moving plasma structures, and apply the so-called stationary wave approach (see Karpman, 1973). It is supposed further that the only variable is $S=x-V t+h z$, where $z$ is along the magnetic field, $x$ is perpendicular to the magnetic field in the direction of the electron drift velocity due to external field, $V$ is the parameter with the velocity dimension (the velocity of the structure) which will be defined from the conditions for the solution existence. For the one-dimensional problem considered in this section, $h=0$, while for the two- and three-dimensional models, $h$ defines the angle of the plane in which the nonlinear structure can move, with the plane perpendicular to the magnetic field, $\vec{B}_{o}$, i.e., the aspect angle for auroral radar reflections (see Sect. 4). Thus, a class of stationary solutions is sought which describes stationary electrostatic structures moving with a constant velocity $V$ in respect to the plasma.

With these suppositions, we derive from the system of equations Eqs. 1-3 the nonlinear equations of motion for electrons and ions. Taking into account the nonlinearity, electron and ion inertia, diffusion, and collisions, the equation can be derived which relates the electron number density to the electric potential $\varphi$ :

$$
\begin{aligned}
& \frac{d^{2}}{d S^{2}}\left(\frac{\tilde{V}_{e}^{2}}{2 N_{e}^{2}}+U_{T e}^{2} \ln N_{e}-\frac{e \varphi}{m_{e}}\right)-\frac{v_{e}}{\tilde{V}_{e}} \cdot \frac{d}{d S}\left(\frac{2 \tilde{V}_{e}^{2}}{N_{e}}+U_{T e}^{2} N_{e}\right) \\
& +\frac{N_{e} v_{e} e}{\tilde{V}_{e} m_{e}} \cdot \frac{d \varphi}{d S}+\left(1-N_{e}\right) \omega_{H e}^{2}=0
\end{aligned}
$$

where $N_{e}=n_{e} / n_{o} ; \tilde{V}_{e}=V\left(1-\delta_{e}\right) ; \delta_{e}=U_{d e} / V ; U_{d e}$ is the electron drift velocity $\left|U_{d e}\right|=|\vec{E}| /|\vec{B}|$.

Neglecting nonlinearity and electron inertia in Eq. 1 we arrive at a simplified equation for the electrons:

$\frac{d}{d S}\left(U_{T e}^{2} \ln N_{e}-\frac{e \varphi}{m_{e}}\right)+\frac{\omega_{H e}^{2} \tilde{V}_{e}}{v_{e}}\left(1-\frac{1}{N_{e}}\right)=0$.

Equation 5 will be further used in the simplified models which consider two-dimensional effects.

For the nonmagnetized ions with the account for nonlinearity, diffusion, collisions, viscosity, and effects of pressure by high-frequency waves (for example, lower-hydrid waves and/or ion plasma waves), the equation relating ion number density $N_{i}$ to the electric potential $\varphi$ will be:

$$
\begin{aligned}
& \frac{d}{d S}\left(\frac{\tilde{V}_{i}^{2}}{2 N_{i}^{2}}+U_{T i}^{2} \ln N_{i}+\frac{e_{i} \varphi}{m_{i}}\right)+\tilde{V}_{i} \eta_{i}\left(1-\frac{1}{N_{i}}\right) \\
& \quad-\eta_{i} \tilde{V}_{i} \frac{d^{2}}{d S^{2}} \cdot\left(\frac{1}{N_{i}}\right)=0 ;
\end{aligned}
$$

where $\tilde{V}_{i}=V\left(1-\delta_{i}\right) ; \delta_{i}=U_{d i} / V ; N_{i}=n_{i} / n_{o i}$. While for the $\mathrm{E}$ region $\delta_{i} \approx 0$, in the upper $\mathrm{E}$ region and higher $\delta_{i}$ is significant and needs to be taken into account for the general theory. Similar equations can be written for different sorts of ions.

The Poisson equation (Eq. 3) for the electric potential $\varphi$ in case of one sort of ions (which only will be considered here) can be written in the form

$N_{e}-N_{i}=\frac{e}{m_{i} \omega_{o i}^{2}} \cdot \frac{d^{2} \varphi}{d S^{2}} ;$

where $\omega_{o i}$ is the ion plasma frequency, $\omega_{o i}^{2}=e^{2} n_{o} / \varepsilon_{o} m_{i}$.

From Eqs. 4, 5 (for electrons), and 6 (for ions), together with the Poisson Eq. 7, a system can be constructed for one-dimensional electrostatic plasma structures in a collisional plasma. The role of particular terms will now be considered by simplifying the problem by neglecting one or some of these effects.

\subsection{The role of ion viscosity}

Let us first consider the role of the ion viscosity using Eq. 4 for electrons and Eq. 6 for ions, but supposing quasineutrality $N_{i}=N_{e}=N$. For this case the following equation is derived:

$$
\begin{aligned}
& -\beta \cdot \frac{d^{3}}{d \xi^{3}}\left(\frac{1}{N}\right)+\tilde{\delta}_{e} \cdot \frac{d^{2}}{d \xi^{2}}\left(\frac{M_{o}^{2}}{2 N^{2}}+\ln N\right)+\gamma \cdot \frac{d}{d \xi}\left(\frac{M_{1}^{2}}{N}+N\right) \\
& +\lambda \cdot(1-N)-\alpha_{\eta} \cdot N \frac{d^{2}}{d \xi^{2}}\left(\frac{1}{N}\right)=0
\end{aligned}
$$

where

$$
\begin{aligned}
& M_{o}^{2}=M^{2}\left[\left(1-\delta_{i}\right)^{2}+\frac{m_{e}}{m_{i}}\left(1-\delta_{e}\right)^{2}\right] ; \beta=\eta_{i} \tilde{V}_{i} \tilde{\delta}_{e} \omega_{L H R} / C_{s}^{3} ; \\
& \tilde{\delta}_{e}=\delta_{e}-1 ; \\
& M_{1}^{2}=M^{2}\left[\left(1-\delta_{i}\right)^{2}+2 \frac{m_{e}}{m_{i}}\left(1-\delta_{e}\right)^{2}+\frac{v_{\mathrm{i}}}{v_{e}}\left(1-\delta_{i}\right) \cdot\left(1-\delta_{e}\right)\right]
\end{aligned}
$$


$\gamma=C_{s} v_{e} /\left(V \cdot \omega_{L H R}\right)$

$\alpha_{\eta}=\eta_{i}\left(1-\delta_{i}\right) \cdot v_{e} / C_{s}^{2} ; \quad M=V / C_{\mathrm{s}} ; \quad \lambda=\tilde{\delta}_{e}-R\left(1-\delta_{i}\right) ;$

$\xi=S \cdot \omega_{L H R} / C_{s} ; \omega_{L H R}^{2}=\omega_{H i} \omega_{H e}$

The first term in Eq. 8 describes dispersion effects; the second is due to diffusion, nonlinearity, and inertia of electrons and ions, and defines nonlinear wave damping (increase), as in Volosevich et al. (1995). The third and fourth terms are due to collisions of the charged particles with neutrals, they define the linear increment $\gamma_{L I N}$ of the FB waves. The last term in Eq. 8 describes the wave damping resulting from the ion viscosity.

It can be noted that when the ion viscosity is neglected $\left(\eta_{i}=0\right)$, Eq. 8 coincides with Eq. 12 of Volosevich et al. (1995).

Numerical solutions of Eq. 8 may be sought, but this is beyond the scope of the present paper. Due to multiple parameters that enter, the numerical results will not be easy to analyze.

To illustrate the solutions in the limit of small amplitudes, consider a case $N=1+n, n=\delta n / n_{0} \ll 1$. The terms with the derivatives on $n^{2}$ may then be neglected in comparison with similar terms with the derivatives on $n$. The term $\alpha_{\eta} n\left(d^{2} n / d \xi^{2}\right)$ is small in comparison with the term $2 \gamma M_{1}^{2} n(d n / d \xi)$. Indeed, consider their ratio $r$ :

$r \sim\left|\frac{\alpha_{\eta}}{2 \gamma M_{1}^{2}}\right| \cdot \frac{r_{N L}}{S} \sim \frac{\omega_{L H R}}{2 M v_{i}} \cdot \frac{1}{1+\tau} \cdot \frac{r_{N L}}{S}$.

Here $r_{N L}=C_{s} / \omega_{L H R}$ is a characteristic length scale. For the $\mathrm{E}$ region $C_{s} \sim 370 \mathrm{~m} / \mathrm{s} ; \quad \omega_{L H R} \sim 3.7 \cdot 10^{4} \mathrm{~s}^{-1}$, thus $r_{N L} \sim 1 \mathrm{~cm}$. For VHF radar aurora scatterers, the structures of decimeter and meter scale are interesting; thus their scales $\xi=S / r_{N L} \gg 1$. Consequently, for our purposes $r \ll 1$, and the corresponding term may be neglected. In this case Eq. 8 simplifies significantly and can be written in a "canonical" form

$\beta \frac{\partial^{3} n}{\partial \xi^{3}}-\sigma \frac{\partial^{2} n}{\partial \xi^{2}}-\gamma_{1} \frac{\partial n}{\partial \xi}+\gamma_{2} \frac{\partial n^{2}}{\partial \xi}-\lambda n=0 ;$

where $\sigma=\tilde{\delta}_{e}\left(M_{o}^{2}-1\right)-\alpha_{\eta} ; \gamma_{1}=\gamma\left(M_{1}^{2}-1\right) ; \gamma_{2}=\gamma M_{1}^{2}$. The term with quadratic nonlinearity is retained $\left(\gamma_{2} \neq 0\right)$.

Equation 9 by its form coincides with that derived and analyzed for the one-wave approximation in Rabinovich and Trubetskov (1984), where the effects of dispersion $(\beta \neq 0)$, nonlinearity $(\sigma \neq 0)$, and dissipation $\left(\gamma_{1} \neq 0\right.$, $\left.\gamma_{2} \neq 0\right)$ are included.

To study the effects of the ion viscosity consider a particular case $\lambda=0$ which corresponds to $U_{d e} \approx V(1+R)$ for $\delta_{i}=0$. This case describes waves at the limit of linear stability for the FB instability. Integrating Eq. 9 in this case we come to an equation of a nonlinear damped oscillator with $\xi$ as analogue of time, $n$ a coordinate of the oscillating point:

$\beta \frac{d^{2} n}{d z^{2}}-\sigma \frac{d n}{d z}-\gamma_{1} n+\gamma_{2} n^{2}=$ const.

The equilibrium positions are at $n_{o 1}=0$ and $n_{o 2}=$ $\gamma_{1} / \gamma_{2}$. To analyze the type of these equilibrium states consider the characteristic equation:

$\beta p^{2}-\sigma p-\gamma_{1}+2 \gamma_{2} n_{o 2}=0$.

If $n=\tilde{n}_{o}+\tilde{n}^{\prime} ; \tilde{n}^{\prime} \sim \exp (p z)$, then the $n_{o 1}=0$ is a saddle point, and $n_{o 2}$ a node for $\sigma^{2}>4 \beta \gamma_{1}$, or a focus for $\sigma^{2}<4 \beta \gamma_{1}$.

This means that if the dispersive effects dominate over the dissipative ones (the latter case), quasi-stationary nonlinear oscillations (knoidal waves) are possible. In particular, when $\sigma=0$, and $c=0$ the exact solution is a dissipative soliton:

$n=n_{\max } / \operatorname{ch}^{2}(\xi / D) ; \quad n_{\max }=\frac{3}{2} \gamma_{1} / \gamma_{2} ; \quad D=\left(4\left|\beta / \gamma_{1}\right|\right)^{1 / 2}$.

In the opposite case, when dissipative effects dominate over the dispersion ones $\left(\sigma^{2} \gg 4\left|\beta \gamma_{1}\right|\right)$, a specific structure of the "electrostatic shock wave" type can develop. It is located between the two quasi-stationary states with $n_{o 1} \approx 0$ for $\xi \rightarrow+\infty(t<0)$ and $n_{o 2} \sim \gamma_{1} / \gamma_{2}=M_{o}^{2}-1 / M_{o}^{2}$ for $\xi \rightarrow-\infty(t>0)$, i.e., between the upstream and downstream regions, respectively. Note that $n_{o 2}$ depends on the velocity of the structure (on its Mach number).

Thus, Eq. 8, even in its small amplitude limit Eq. 9, gives a variety of nonlinear moving structures in a collisional plasma. Equation 9 has a canonical form its solutions are illustrated in various books on nonlinear oscillations. With a proper choice of parameters they probably could be relevant for the auroral E-region conditions during weak radar aurora reflections resulting from smallamplitude inhomogenieties.

Numerical analysis of the full equation (Eq. 8) will allow to study various scenarios of the nonlinear wave evolution, not limited to small amplitudes. Such structures are supposed to be effective radar aurora scatterers. Their spatial scales can be small enough to scatter even decimeter waves as observed in radar aurora. However, as will be shown in Sect. 4, the two-dimensional effects are very important for the saturation level of the turbulence in the E region.

\subsection{The role of deviation from quasi-neutrality}

Now consider the effects of a deviation from quasi-neutrality, that is $N_{i} \neq N_{e}$. This is another way for the wave dispersion to appear in the auroral E-region plasma. For this, Eq. 5, for electrons and Eq. 6 for ions together with the Eq. 7 will be used. After some algebra the following simplified equation for the ion density $N=N_{i}$ is derived:

$$
\begin{aligned}
& \gamma \frac{d}{d \xi}\left(\frac{M_{o}^{2}}{N}+N-\frac{M_{3}}{N^{2}}\right)+\lambda(1-N) \\
& -\alpha_{\eta} \cdot N \frac{d^{2}}{d \xi^{2}}\left(\frac{1}{N}\right)+\beta_{\lambda} \frac{d^{2}}{d \xi^{2}}\left(\frac{M_{1}^{2}}{2 N^{2}}+\ln N\right) \\
& -\frac{d^{3}}{d \xi^{3}}\left[\alpha_{\lambda}\left(\frac{M_{1}^{2}}{2 N^{2}}+\ln N\right)-\left(\frac{\alpha_{\lambda \eta}}{N}\right)\right] \\
& +\beta_{\lambda \eta} \frac{d^{4}}{d \xi^{4}}\left(\frac{1}{N}\right)=0,
\end{aligned}
$$


where

$\alpha_{\lambda}=\mu v_{e} \tau / \omega_{L H R}(1+\tau)^{2} \cdot M ; \quad \alpha_{\lambda \eta}=\eta_{i} \mu \omega_{L H R} \cdot \tilde{V}_{i} / C_{s}^{3} ;$

$\beta_{\lambda}=\mu\left(1-\delta_{e}\right) /(1+\tau) ; \quad \beta_{\lambda \eta}=\eta_{i} \mu v_{e} / C_{s}^{2} ; \quad \mu=\omega_{L H R}^{2} / \omega_{o i}^{2} ;$

$M_{3}=\tilde{\delta}_{e} v_{i} V^{2} \mu /\left(C_{s}^{2} v_{e}\right)$.

Here, $\alpha_{\lambda}$ and $\beta_{\lambda}$ are defined by deviation from quasineutrality, while $\alpha_{\lambda \eta}$ and $\beta_{\lambda \eta}$ by dispersion effects due to ion viscosity.

For a small-amplitude case, in analogy to Sect. 3.2, Eq. 12 can be reduced to the canonical form (Eq. 9) but with the new coefficients with index $\lambda$ :

$\beta_{\lambda}=\alpha_{\lambda}\left(M_{1}^{2}-1\right)-\alpha_{\lambda \eta} ; \quad \sigma_{\lambda}=\beta_{\lambda}\left(M_{1}^{2}-1\right)-\alpha_{\lambda} ;$

$\gamma_{1}=\gamma\left(M_{0}^{2}-1-2 M_{3}\right) ; \quad \gamma_{2}=\gamma\left(M_{0}^{2}-3 M_{3}\right)$.

It can be noted that the respective soliton-type solution for $\lambda=\sigma=0$ of the form of Eq. 11 will hold. However, the spatial scale $D_{\lambda \eta}$ of the structure, which is due to deviation from quasi-neutrality, is reduced in comparison with $D$ as given in Eq. 11:

$D_{\lambda \eta} / D \sim\left(\beta_{\lambda} / \beta\right)^{1 / 2} \sim\left(\mu / \delta_{e}\right)^{1 / 2} \sim 0.1$.

To evaluate a relative role of the loss of quasi-neutrality in the wave dispersion (described by the terms with $\alpha_{\lambda}$ and $\left.\alpha_{\lambda \eta}\right)$, the ratios of the coefficients of the dispersion terms in Eqs. 8 and 12 are to be considered:

$r_{1}=\beta / \alpha_{\lambda} \sim M^{2} \omega_{o i}^{2} /\left(v_{i} v_{e}\right) \quad$ and $\quad r_{2}=\beta / \alpha_{\lambda \eta} \sim \tilde{\delta}_{e} \omega_{e i}^{2} / \omega_{L H R}^{2}$.

The ratios $r_{1}, r_{2}$ are small; that is, the loss of quasineutrality is important for the dispersive properties of a collisional plasma, only at low plasma densities when $\omega_{o i}^{2} \sim \omega_{L H R}^{2}$. Evaluating $r_{1}, r_{2}$ for plasma parameters characteristic for the auroral $\mathrm{E}$ region, it is seen that such conditions are rare. This is in accordance with the conclusions of Gurevich et al. (1994).

It can be concluded that the ion viscosity is the most important process that determines the existence of quasistationary plasma structures of finite amplitude in the collisional auroral E region. It makes the waves dispersive, and this opposes the wave front steepening and overturn. It has already been noted that according to Volosevich (1978) this viscosity is actually a result of the ion Landau damping for large $k$-values.

\section{A two-dimensional model}

From the simplified Eq. 5 for electrons, and neglecting nonlinearity and ion inertia, and for the quasi-neutrality condition $N_{e}=N_{i}$, the two-dimensional equation for electrons is now derived:

$$
\begin{aligned}
\frac{\partial n}{\partial t} & +V_{o e} \nabla n-\frac{v_{e} U_{T e}^{2}}{\omega_{H e}^{2}}\left(\Delta_{\perp} n+\frac{\omega_{H e}^{2}}{v_{e}^{2}} \frac{\partial^{2} n}{\partial Z^{2}}\right) \\
& +\frac{e v_{e}}{m_{e} \omega_{H e}^{2}}\left[n\left(\Delta_{\perp} \varphi+\frac{\omega_{H e}^{2}}{v_{e}^{2}} \frac{\partial^{2} \varphi}{\partial Z^{2}}\right)+\nabla_{\perp} n \cdot \nabla_{\perp} \varphi\right. \\
& \left.+\frac{\omega_{H e}^{2}}{v_{e}^{2}} \frac{\partial n}{\partial Z} \cdot \frac{\partial \varphi}{\partial Z}\right]-\frac{1}{B}[\nabla n, \nabla \varphi]_{z}=0 ;
\end{aligned}
$$

where:

$\Delta_{\perp}=\frac{\partial^{2}}{\partial x^{2}}+\frac{\partial^{2}}{\partial y^{2}} ; \quad \nabla_{\perp}=\frac{\partial}{\partial x} \vec{i}+\frac{\partial}{\partial y} \vec{j} ; \quad n=\delta n / n_{o}$.

The last term in Eq. 13, the so-called vector nonlinearity, describes the main two-dimensional effect in the plane orthogonal to magnetic field $\vec{B}_{o}$. The dependence on the coordinate $Z$ (along magnetic field) can be neglected if $|\partial / \partial Z| \ll\left(v_{e} / \omega_{H e}\right)\left|\partial / \partial r_{\perp}\right|$, that is, when the characteristic length along the magnetic field $L_{z}$ is much larger than the perpendicular one, $L_{\perp}$ (or, more precisely, when $\left.L_{z}^{2} \gg L_{\perp}^{2} v_{e}^{2} / \omega_{H e}^{2}\right)$.

The ion inertia can be accounted for by introducing the operator:

$v_{i} \rightarrow \hat{v}_{i}=v_{i}\left(1+\frac{1}{v_{i}} \frac{\partial}{\partial t}\right)\left(1+\frac{\omega_{H i}^{2}}{v_{i}^{2}}\right)$.

Then the equation for ions will be:

$\hat{v}_{i} \frac{\partial n}{\partial t}-\left(\frac{e}{m_{i}}\right) \nabla(n \nabla \varphi)-U_{T i}^{2} \Delta n-\left(\frac{\omega_{H i}^{2}}{v_{i}^{2}}\right) \frac{1}{B}[\nabla n, \nabla \varphi]_{z}=0$.

For nonmagnetized ions using Eq. 13 for electrons, the equation for the charged particle density $n$ is derived for the two-dimensional case $\partial / \partial Z=0$ :

$$
\begin{aligned}
(1+\mathrm{R}) & \frac{\partial n}{\partial t}+U_{e o} \nabla n+\frac{R}{v_{i}}\left(\frac{\partial^{2} n}{\partial t^{2}}-C_{s}^{2} \Delta_{\perp} n\right) \\
& +\frac{1}{B}[\nabla n, \nabla \varphi]_{z}=0 .
\end{aligned}
$$

It is seen in Eq. 15, that its first two terms describe the linear dispersion law for the FB instability $\omega \sim$ $\vec{k} \vec{V}_{o e} /(1+R)$; the third term is a linear increment of the perturbations $\quad \gamma_{L I N}=R\left(\omega^{2}-k^{2} C_{S}^{2}\right) / v_{i}$ for $n \sim$ $n_{o} \exp i(\omega t-k r)$. The last term, the vector nonlinearity, describes the nonlinear wave spreading in the plane perpendicular to the magnetic field. This term in some conditions can be the dominant one in the evolution.

The nonlinear wave-wave interactions were studied in detail within the frame of the weak turbulence theory. However, as was mentioned in Sect. 1, it is applicable only to weak nonlinear interactions characteristic to small wave amplitudes. In contrast, the two-dimensional theory described, must be applicable to finite amplitude waves and strong wave-wave interactions leading to wave spreading in the plane perpendicular to the magnetic field. This wave spreading is capable, in principal, of leading to the stabilization, i.e., to a stationary turbulence level.

The dependence on the $z$ coordinate is most important for the magnetized electrons. In the simplest case, instead of Eqs. 5 and 13 we can have equations where the electron collision frequency $v_{e}$ is replaced by $v_{e}\left[1+h^{2}\left(\omega_{H e}^{2} / v_{e}^{2}\right)\right]$. For large aspect angles, or for $h^{2} \gg v_{e}^{2} / \omega_{H e}^{2}$, it follows from the linear theory that the intensity of nonlinear stationary waves becomes negligibly small. In the opposite case, or for small aspect angles $h^{2} \ll v_{e}^{2} / \omega_{H e}^{2}$, the corrections are expected to be small. However, a proper analysis of the respective three-dimensional problem is beyond the scope of the present paper. 
For illustrative purposes we show such stabilization by the two-dimensional wave spreading on a simple example. Suppose that the wave $n_{1}$ is due to the electron drift with the velocity $U_{d x}$ in the $X$ direction. Then the electric field of this wave $E_{1}=-\partial \varphi_{1} / \partial x$ induces the drift of the electrons in the $Y$ direction with the velocity

$\left|U_{e y}\right|=\frac{1}{B} \frac{\partial \varphi}{\partial x}=\left(v_{i} / \omega_{H i}\right)\left|U_{d x}\right| n_{1}$.

It is clear that an additional wave $n_{2}$ is created, which propagates in the $Y$ direction. It means that the electric potential $\varphi$ now depends also on the $Y$ coordinate. Now, supposing that $n_{1} \sim 0.1$ and $v_{i} / \omega_{H i} \sim 10$, it is seen that amplitudes $n_{1}$ and $n_{2}$ are of comparable amplitude at this level of the auroral E-region ionosphere.

From Eq. 15 the equations for the two waves moving in orthogonal directions are:

$$
\begin{gathered}
\frac{\partial n_{1}}{\partial t}(1+R)+U_{e 0 x} \nabla_{x} n_{1}+\frac{R}{v_{1}}\left(\frac{\partial^{2} n_{1}}{\partial t^{2}}-C_{s}^{2} \Delta_{\perp} n_{1}\right) \\
+\frac{1}{B}\left(\frac{\partial n_{2}}{\partial x} \frac{\partial \varphi_{1}}{\partial y}-\frac{\partial n_{1}}{\partial y} \frac{\partial \varphi_{2}}{\partial x}\right)=0, \\
\frac{\partial n_{2}}{\partial t}(1+R)+U_{e y} \nabla_{y} n_{2}+\frac{R}{v_{1}}\left(\frac{\partial^{2} n_{2}}{\partial t^{2}}-C_{s}^{2} \Delta_{\perp} n_{2}\right) \\
+\frac{1}{B}\left(\frac{\partial n_{2}}{\partial y} \frac{\partial \varphi_{1}}{\partial x}-\frac{\partial n_{1}}{\partial x} \frac{\partial \varphi_{2}}{\partial y}\right)=0 .
\end{gathered}
$$

It may be seen that in a small amplitude limit both waves are nonlinear. Using for simplicity a linear relation between $n_{1}, n_{2}$, and electric potential $\varphi$, and retaining nonlinear terms, we obtain the equation for the wave $n_{1}$ :

$$
\begin{gathered}
\frac{\partial n_{1}}{\partial t}(1+R)+\frac{R}{v_{i}}\left(\frac{\partial^{2} n_{1}}{\partial t^{2}}-C_{s}^{2} \Delta_{\perp} n_{1}\right)+U_{e o x} \frac{\partial n_{1}}{\partial x} \\
+\beta U_{e x} n_{2} \frac{\partial n_{1}}{\partial y}-\beta^{2} U_{e x} n_{1} \frac{\partial\left(n_{1} n_{2}\right)}{\partial x}=0 .
\end{gathered}
$$

The first three terms of Eq. 17 describe the linear stage of the instability, the fourth term the transfer by the wave $n_{2}$ of the wave $n_{1}$. Most important is the last term, which describes the modification of the wave $n_{1}$ which takes place due to the wave $n_{2}$. This is just the process which limits the linear wave growth for the waves propagating in the plane perpendicular to the magnetic field.

This process can be considered approximately when $\left(\partial n_{2} / \partial x\right) \ll\left(\partial n_{2} / \partial y\right)$ and $\left(\partial n_{1} / \partial y\right) \ll\left(\partial n_{1} / \partial n\right)$, by introducing again a stationary-wave approach. Then from Eq. 17 we have

$\frac{\partial n_{1}}{\partial S}\left(\delta_{e}-(1+R)\right)+\frac{R C_{s}^{2}\left(M^{2}-1\right)}{V v_{i}} \frac{\partial^{2} n_{1}}{\partial S^{2}}-\beta^{2} \delta_{e} n_{2} \frac{\partial n_{1}^{2}}{\partial S}=0$.

Its solution can be written in a simple form:

$n_{1} \sim \tilde{\delta}_{e} /\left(\tilde{\beta}-e^{\theta S}\right)$,

where $\delta_{e}=U_{d e} / V ; \quad s=x-V t ; \quad \theta=R C_{s}^{2} /\left(v_{i} \cdot \delta_{e} \cdot V\right) ; \tilde{\delta}_{e}=$ $\delta_{e}-(1+R) ; n=\delta n / n_{o} ; \quad \widetilde{\beta}=\beta^{2} \cdot n_{2} \cdot \delta_{e} / 2 ; \beta=v_{i} / \omega_{H i}$.
A stationary state $n \rightarrow 0$ exists for $S \rightarrow+\infty \quad(t<0)$, while for $S \rightarrow-\infty \quad(t>0)$ a stationary turbulence level can be established at

$n^{*}=\tilde{\delta}_{e} \tilde{\beta}=2 \tilde{\delta}_{e} /\left(\beta^{2} \delta_{e} \cdot n_{2}\right)$.

It can be seen that the stationary level only weakly depends on the degree of the supercriticality when $\delta_{e} \gg 1+R$. It is defined mainly by the parameter $\left(v_{i} / \omega_{n i}\right)^{2}$ and is inversely proportional to the amplitude $n_{2}$. If we suppose that $n_{1} \sim n_{2}$, then $n^{*} \sim \sqrt{2} \omega_{H i} / v_{i}$, and the density fluctuation amplitude for the $\mathrm{E}$ region amounts to about $10 \%$, the value deduced from observations.

\section{Discussion and possibilities for comparisons with experiments}

After the discovery of the radar aurora, magnetospheric and auroral researchers were excited by the perspective to be able to perform a continuous registration of auroral phenomena irrespective of weather and solar-illumination conditions. Radar aurora was extensively studied during IGY (1957-1958) and further on. These expectations were strengthened when a successful interpretation of the main features of this phenomenon was achieved as a result of the modified two-stream, or FB, plasma instability in the auroral E region [see Kelley (1989) for a comprehensive review and references].

Studies of auroral plasma inhomogenieties which can be auroral VHF scatterers, were performed by remote radiophysical methods and by in situ measurements from rockets. They revealed the extreme complexity of the phenomenon, its nonlinearity and existence of different instability modes in different conditions. Significant localized electron heating was found in the scattering region, implying nonlinear wave-wave and wave-particle interactions including Landau damping. These results led to an extensive theoretical research of the radar aurora [for recent analyses and reviews see Hamza and St.-Maurice (1995), Gurevich et al. (1994), and Dimant and Sudan (1995)]. However, their exact physical nature, motions, waveforms, and lifetimes are still unclear and remain a subject of intense study.

Studies of radar aurora are having a new start with the advent of unprecedented global magnetosphericionospheric coordinated experiments in 1995-1998 from several recently launched high-altitude magnetospheric satellites, including GEOTAIL, WIND, POLAR, INTERBALL-1 and, being prepared for launching, INTERBALL-2 (see Galeev et al., 1995; Galperin and Zelenyi, 1995), and CLUSTER, together with a network of ground-based geophysical stations. Development and extension of the EISCAT incoherent radars, of the SuperDARN global HF radar network, and of various other $\mathrm{HF}$ and VHF radar systems give a new perspective in the study and geophysical interpretation of the auroral plasma inhomogenieties.

What particular geophysical parameter(s) can be deduced from the VHF radar aurora observations with medium and high space and time resolution? What are 
particular constraints on the data interpretation in terms of deduced geophysical parameters which are imposed by the location of the observational site (or magnetic aspect angle), by imprecise knowledge of the reflection altitude, collision frequencies, and of electric-field gradients? There is still no adequate nonlinear theory to answer these important and practical questions raised by experimenters.

An attempt in this direction is presented in this paper. As was shown in Volosevich et al. (1995) there are theoretical reasons to believe that some types of MHD nonlinear soliton-like plasma structures with scales in decimeter and meter ranges which are moving with velocities of order of the ion sound speed could be responsible for at least some types of the radar aurora observed. An extension of this theory is presented here. However, such stationary electrostatic MHD structures moving with constant velocity can by no means exhaust the plethora of plasma phenomena on auroral field lines at various altitudes and geophysical conditions, and much further work, especially an extension to a three-dimensional theory is necessary.

The problem of stability of the solutions/structures can be considered if we suppose that the linear dispersion relation shown in Sect. 1 is modified during the nonlinear stage in such a way that the nonlinear frequency $\omega_{N L}$ becomes a function $\omega_{N L}=\omega\left(k, A^{2}\right)$, where $A$ is the wave amplitude. For the nonlinear theories of FB instability (see, for example, Volosevich and Liperovsky, 1975; Sudan, 1983; Hamza and St.-Maurice, 1995) it follows that $\omega_{N L}$ is nearly proportional to $A^{-2}$, so that the dispersion relation has the form:

$$
\begin{gathered}
\omega=\frac{\vec{k} \cdot \vec{V}_{o e}}{1+\hat{R}\left(1+g^{2} \frac{k^{2} U_{T i}^{2}}{v_{i}^{2}}+\alpha_{N L} A^{2}\right)} ; \\
\hat{R}=\frac{v_{i} v_{e}}{\omega_{H i} \cdot \omega_{H e}} \cdot\left(1+\frac{k_{\|}^{2}}{k_{\perp}^{2}} \cdot \frac{\omega_{H e}^{2}}{v_{e}^{2}}\right),
\end{gathered}
$$

and thus $\left(d \omega / d A^{2}\right)_{A \rightarrow 0}<0$. Thus, inclusion of the ion viscosity term in Eqs. 8 and 9 leads then to $\left(d^{2} \omega / d^{2} k\right)_{k=k_{0}, A \rightarrow 0}<0$. Using the stability criteria for stationary waves against disturbances propagating in the same direction, as described by Lighthill (1965), it can be concluded that a one-dimensional stationary wave will be stable. However, for a stability analysis in a two-dimensional model a more precise knowledge is needed about the nonlinear dispersion relation, probably with the account of the multi-ion composition and currents in the auroral E region. From an observational point of view it seems that various instability regimes depending on local parameters of the medium, especially changing electric fields and currents, have an important role in the physical, not mathematical, stability or intermittence of the observable small-scale plasma structures in the auroral E region.

It is rather difficult to compare the theory described here with available experimental data. The main part of experimental information about auroral VHF-radar aurora is gathered by remote radio methods. Such measurements well characterize the average scattering properties of the medium, its bulk velocity, and various morphological types of reflections that are observed (see, Schlegel et al., 1990; Villain et al., 1990; Foster et al., 1992; Haldoupis et al., 1992, 1995). However, the impulse length in these experiments was not less than $0.3 \mathrm{~km}$ and often much more, and azimuthal width was even more. Hence many scattering centers took part in the formation of the return signal. So, their waveforms and other microscopic characteristics important for comparisons with the theory could hardly be assessed.

Meanwhile these data, and especially some results of well-instrumented rocket experiments, revealed the strongly nonlinear nature of the plasma in the collisional auroral E region. For example, in the experiment by Bahnsen et al. (1978), after its reinterpretation in Primdahl and Bahnsen (1985), a good agreement was found with the theory by Sudan (1983). It was achieved when the effective electron collision frequency was considered to be an order of magnitude higher than that due to electron-neutral collisions. It indicated strong wave-particle interactions resulting in a significant electron heating. Important electron heating and anomalous collision frequencies deduced from EISCAT and rocket data could indicate effects of electron Landau damping through the modulational instability (Stenflo, 1991). Recent particle-in-cell simulations by Schlegel and Thiemann (1994) succeeded in reproducing the observed electron heating, its dependence on the ratio $U_{d e} / U_{T i}$ (in our designations), and several other observational characteristics. However, the three-dimensional nature of these effects is beyond the scope of the present paper.

The other, most important result of the rocket experiments performed, is an evaluation of the electron-density amplitude variation, which appeared to be as high as 5-15\% (Kelley and Mozer, 1973; Primdahl and Bahnsen, 1985, and references therein). It indicated that a strong turbulence theory is needed for its description. As was demonstrated by a simplified example in Sect. 4, these high amplitudes must lead to strong nonlinear wave spreading and other effects related to the two-dimensional turbulence-level stabilization. It was shown in Sect. 4 that this level can be of order of $10 \%$ in density, as observed.

We are not aware of published direct waveform data in the range of decimeter and meter wavelengths in the auroral $\mathrm{E}$ region, in conditions of saturated $\mathrm{FB}$ instability, which could be compared with those predicted by the theory. Thus, no definite comparisons of the experimental rocket data on waveforms with those predicted by the theory have been made so far. As can be seen from Eq. 8, the waveforms depend on many ambient parameters of the medium, sometimes not all of them are known. Thus, such comparisons need particular care in each case.

However, some indirect information about waveforms can be extracted from extensive statistical analyses of the electric-field data of two rocket experiments in the saturated FB instability conditions by Pecseli et al. (1989, 1993). In one experiment with a low turbulence level, large coherent structures (bursts or wave packets) were not detected. However, the waves were found with a velocity 
significantly lower than the ion sound speed, and deviated from the $\boldsymbol{E} \times \boldsymbol{B}$ direction to that consistent with $\hat{R}=$ const. While inconsistent with the linear theory, such wave properties can be qualitatively accommodated in the theory presented here by a proper choice of parameters.

In the other experiment, when the turbulence level was higher, an elaborated statistical analysis definitely indicated the presence of phase relations between the Fourier harmonics, or in other words, significant nonlinear couplings. While no waveforms were presented, these results, in our opinion, do not seem to contradict the existence of some characteristic waveforms, or even solitons, in the measured signals. These results probably deserve some further analysis by the wavelet technique based on model waveforms derived from the solutions of Eqs. 8 or 9.

It can be noted that in the collisionless limit of this theory (Volosevich and Galperin, 1995) the predictions made are in reasonable, though qualitative, agreement with the in situ observations of double layers and ion holes on auroral field lines at high altitudes.

It is hoped that the amplitudes, motions, waveforms, and lifetimes of the nonlinear plasma structures in the $\mathrm{E}$ and $\mathrm{F}$ regions in various geophysical conditions will be possible to predict within the frame of the theory presented, its further extensions to $3 \mathrm{D}$ and by its numerical modeling. If these predictions are successful, then a new endeavor in the auroral and magnetospheric real-time medium-scale and large-scale diagnostics will become possible from the VHF-radar aurora observations. However, much further work is needed to achieve this goal, including three-dimensional extension and critical experimental tests of the theory together with extensive numerical modeling.

\section{Conclusions}

1 A nonlinear equation (Eq. 8) is derived which describes the evolution of stationary electrostatic waves of arbitrary amplitude in a one-dimensional collisional MHD model.

2 An evaluation is made of the influence of the linear wave dispersion, collisions, nonlinearity, electron and ion inertia, and ion viscosity for conditions characteristic of the auroral E-region ionosphere.

3 It is shown that for certain FB wave-growth conditions a wave overturn of the wave front occurs for nondispersive waves.

4 Small dispersive corrections to the dispersion equation for the FB waves are shown to be of principal importance for stationary solutions.

$5 \mathrm{It}$ is shown that the ion nonlinear and inertial effects for realistic ionospheric conditions are more important than those for electrons.

6 It is shown that for a two-dimensional model the main nonlinear process which leads to a turbulent level stabilization is the process of the two-dimensional wave spreading in the plane perpendicular to the magnetic field. This wave transfer is described by the term with the vector nonlinearity, or the Poisson brackets (see Eq.
13). The wave transfer can proceed to the region of lower $k$-values (larger scales).

7 The resulting two-dimensional structures can be stable in a narrow range of the parameter space $\left(N_{i}, m_{i}, V_{\alpha}, V, v_{i} / \omega_{H i}, v_{e} / \omega_{H e}\right)$. The steady-state amplitude level is mainly determined by the ionosphere factor $v_{i} / \omega_{H i}$, and thus is altitude dependent.

8 The theoretical model considered allows to describe various evolution scenarios of the plasma instability in a collisional auroral E-region plasma which can include the conditions of a radar aurora.

Acknowledgments. This work was performed with the support of the INTAS grant 95-1695 and of the Russian Foundation for Basic Research grant 94-02-04299.

Topical Editor, D. Alcaydé thanks A. M. Hamza and H. Pécseli for their help in evaluating this paper.

\section{References}

Arnoldy, R. L., K. A. Lynch, P. M. Kintner, and J. L. Vago, Smallscale structure of auroral electron and ion acceleration, in Physics of Space Plasmas, Eds. T. Chang and J. R. Jasperse, Scientific Publishers, Cambridge, Mass., pp. 73-96, 1992.

Bahnsen, A., E. Ungstrup, C.-G. Falthammar, U. Fahleson, J. K. Olesen, F. Primdahl, F. Spangslev, and A. Pedersen, Electrostatic waves observed in an unstable polar cap ionosphere, J. Geophys. Res., 83, 5191-5197, 1978.

Boehm, M. H., C. W. Carlson, J. P. McFadden, and F. S. Mozer, Observations of double layer-like and soliton-like structures in the ionosphere, Geophys. Res. Lett., 11, 511-514, 1984.

Braginsky, S. I., Transport phenomena in plasma, in Problems of Plasma Theory, Ed. M. L. Leontovich, Gosatomizdat, Moscow, pp 183-272, 1963.

Bryant, D. A., A. C. Cook, Z.-S. Wang, U. de Angelis, and C. H. Perry, Turbulent acceleration of auroral electrons, J. Geophys. Res., 96, 13829-13839, 1991.

Chang, T. S., Exact nonlinear EIC waves of arbitrary amplitude and cavitons, Int. J. Eng. Sci., 30, N 2, 231-236, 1982.

Chang, T. S., Lower-hybrid collapse, caviton turbulence, and charged particle energization in the topside auroral ionosphere and magnetosphere, Phys. Fluids B, 5(7), 2646-2656, 1992.

Dimant, Y. S., and R. N. Sudan, Kinetic theory of Farley-Buneman instability in the E region of the ionosphere, J. Geophys. Res., 100, 14605-14623, 1995.

Erlandson, R. E., L. J. Zanetti, M. H. Acuna, A. I. Eriksson, M. H. Boehm, and L. G. Blomberg, Freja observations of electromagnetic ion cyclotron ELF waves and transverse ion acceleration on auroral field lines, Geophys. Res. Lett., 21, 1855-1858, 1994.

Fejer, B. G., and M. C. Kelley, Ionospheric irregularities, Rev. Geophys. Space Phys., 18, 401, 1980.

Foster, J. C., D. Tetenbaum, C. F. del Pozo, J. P. St-Maurice, and D. R. Moorcroft, Aspect angle variations in intensity, phase velocity, and altitude for high-latitude $34-\mathrm{cm}$ E-region irregularities, J. Geophys. Res., 97, 8601-8617, 1992.

Galeev, A. A., Yu. I. Galperin, and L. M. Zelenyi, The INTERBALL Project to study solar-terrestrial physics, in Interball Mission and Payload, Eds. Yu. Galperin, T. Muliarchik, and J.-P. Thouvenin, RKA, IKI, CNES, Paris, pp 11-45, 1995.

Galperin, Yu. I., and L. M. Zelenyi, INTERBALL mission strategy and recent results in IKI on some of the mission oriented theory directions, in Proceedings of the CLUSTER Workshop on Physical Measurements and Mission Oriented Theory, Ed. C. Mattok, ESA SP-371, Noordwijk, pp. 187-195, 1995.

Gershman, B. N., Yu. A. Ignatiev, and G. H. Kamenetskaya, Formation Mechanisms of Ionospheric Sporadic Es Layer at Various Latitudes (in Russian), Nauka, Moscow, p. 108, 1976. 
Gurevich, A. V., N. D. Borisov, and K. P. Zybin, Ionospheric Eregion turbulence induced by the turbulence of neutral atmosphere, Preprint MPAE-W, 100-95-02, 2-35, 1994.

Haldoupis, C. A., A review on radio studies of auroral E-region ionospheric irregularities, Ann. Geophysicae, 7, 239, 1989.

Haldoupis, C. A., On type 3 auroral VHF coherent radar backscatter, J. Geophys. Res., 97, 4109-4120, 1992.

Haldoupis, C. A., J. A. Koehler, G. J. Sofko, D. W. Danskin, D. Andre, and $\mathbf{J}$. Mu, Localized and strongly unstable plasma regions in the auroral $\mathrm{E}$ region ionosphere and implications for radar experiments, J. Geophys. Res., 100, 7771-7782, 1995.

Hamza, A. M., and J. P. St.-Maurice, A fully self-consistent fluid theory of anomalous transport in FD turbulence, J. Geophys. Res., 100, 9653-9668, 1995.

Karpman, V. I., Nonlinear Waves in Dispersive Media (in Russian), Nauka, Moscow, p. 175, 1973.

Kelley, M. C., The Earth's Ionosphere: Plasma Physics and Electrodynamics, Academic Press, San Diego, 1989.

Kelley, M. C., and F. S. Mozer, Electric field and plasma density oscillations due to the high-frequency Hall current two-stream instability in the auroral E region, J. Geophys. Res., 78, 2214-2221, 1973.

Kintner, P. M., J. L. Vago, S. W. Chesney, R. L. Arnoldy, K. A Lynch, C. J. Pollock, and T. E. Moore, Localised lower hybrid acceleration of ionospheric plasma, Phys. Rev. Lett., 68, 2448-2451, 1992.

Koskinen, H., R. Lundin, and B. Holback, On the plasma environment of solitary waves and weak double layers, J. Geophys. Res., 95, 5921-5929, 1990

LaBelle, J., P. M. Kintner, A. W. Yau, and B. A. Whalen, Largeamplitude wave packets observed in the ionosphere in association with transverse ion acceleration, J. Geophys. Res., 91, 7113-7118, 1986.

Lighthill, M. J., Contributions to the theory of waves in nonlinear dispersive systems, J. Inst. Math. Appl., 1, 269, 1965.

McFadden, J. P., C. W. Carlson, and M. H. Boehm, Field-aligned electron precipitation at the edge of an arc, J. Geophys. Res., 91, 1723-1730, 1986.

O'Brien, B. J., and D. L. Reasoner, Measurements of highly collimated short-duration bursts of auroral electrons and comparison with existing auroral models, J. Geophys. Res., 76, 8258, 1971

Pecseli, H. L., F. Primdahl, and A. Bahnsen, Low-frequency electrostatic turbulence in the polar-cap E region, J. Geophys. Res., 94, $5337-5349,1989$.

Pecseli, H. L., J. Truslen, A. Bahnsen, and F. Primdahl, Propagation and nonlinear interaction of low-frequency electrostatic waves in the polar-cap E region, J. Geophys. Res., 98, 1603-1612, 1993.

Primdahl, F., and A. Bahnsen, Auroral E-region diagnosis by means of non-linearly stabilised plasma waves, Ann. Geophysicae, 3, $57-62,1985$.

Rabinovch, M. I., and D. I. Trubetskov, Introduction to the theory of oscillations and waves, Nauka, Moscow, pp 432, 1984.
Reme, H., and J.-M. Bosqued, Evidence near the auroral ionosphere of a parallel electric field deduced from energy and angular distributions of low-energy particles, J. Geophys. Res., 76, 7683, 1971.

Rinnert, K., Plasma waves observed in the auroral E-region during ROSE campaign, J. Atmos. Terr. Phys., 54, 683, 1992.

Romero, H., G. Ganguli, and Y. C. Lee, Ion acceleration and coherent structures generated by lower hybrid shear-driven instabilities, Phys. Rev. Lett., 69, N 24, 3503-3506, 1992.

Schamel, H., Electron holes, ion holes, and double layers: Electrostatic phase space structures in theory and experiments, Phys. Rep., 140, 161-191, 1986.

Schlegel, K., and H. Thiemann, Particle-in-cell plasma simulations of the modified two-stream instability, Ann. Geophysicae, 12, 1091-1100, 1994

Schlegel, K., T. Turunen, and D. R. Moorcroft, Auroral radar measurements at $16-\mathrm{cm}$ wavelength with high range and time resolution, J. Geophys. Res., 95, 19001-19009, 1990.

Seyler, C. E., A mathematical model of the structure and evolution of small-scale discrete auroral arcs, J. Geophys. Res., 95, 17199-17315, 1990.

Stenflo, L., Wave collapse in the lower part of the ionosphere, J. Plasma Phys., 45(3), 355-359, 1991.

Sudan, R. N., Unified theory of type I and type II irregularities in the equatorial electrojet, J. Geophys. Res., 88, 4853-4860, 1983.

Temerin, M., M. Woldorff, and F. S. Mozer, Nonlinear steepening of the electrostatic ion cyclotron wave, Phys. Rev. Lett., 43, 1941-1943, 1979

Temerin, M., C. A. Cattell, R. L. Lysak, M. K. Hudson, R. B. Torbert, F. S. Mozer, R. D. Sharp, and P. M. Kintner, The small-scale structure of electrostatic shocks, J. Geophys. Res., 86, 11278-11298, 1981.

Tetreault, D. J., Theory of electric fields in the auroral acceleration region, J. Geophys. Res., 96, 3549-3563, 1991.

Villain, J.-P., C. Hanuise, R. A. Greenwald, K. B. Baker, and J. M. Ruohoniemi, Obliquely propagating ion acoustic waves in the auroral $\mathrm{E}$ region: Further evidence of irregularity production by field-aligned electron streaming, J. Geophys. Res., 95, 7833-7846, 1990.

Volosevich, A. V., Farley-Buneman instability in the polar ionosphere, in Phenomena in Polar Ionosphere, Ed. G. A. Loghinov (in Russian), Nauka, Leningrad, pp. 50-61, 1978.

Volosevich, A. V., and Yu. I. Galperin, Non-linear quasi-stationary electrostatic structures in magnetospheric plasma, Cosmic Res. (English translation), 33, 48-56, 1995.

Volosevich, A. V., and V. A. Liperovsky, Generation of small-scale inhomogenieties in a turbulent plasma and radar aurora, Geomagn. Aeron. (in Russian), 15, 74-77, 1975.

Volosevich, A. V., Yu. I. Galperin, and A. G. Sugakevich, Non-linear quasi-stationary electrostatic waves and solitonlike structures in collisional polar E-region plasma, Cosmic Res. (English translation), 33, 57-63, 1995.

Whalen, B. A., and P. W. Daly, Do field-aligned auroral particle distributions imply acceleration by quasi-static parallel electric fields? J. Geophys. Res., 84, 4175-4182, 1979. 